Rev. High Pressure Sci. Technol., Vol. 7 (1998) 157 159

\title{
First-Principles Calculations of Hyperfine Parameters of Solid Iodine under High Pressure
}

\author{
Kenji Yamaguchi* and Hiroshi Miyagi \\ Department of Material Physics, Faculty of Engineering Science, \\ Osaka University, Toyonaka, Osaka 560, Japan
}

\begin{abstract}
Using the full-potential linear muffin-tin-orbital method, we study hyperfine parameters of solid iodine under high pressure theoretically. Above $15 \mathrm{GPa}$, the direction of principal component of the electric field gradient (EFG) suddenly changes as reported by Mössbauer experiments. In the monatomic phase, the large asymmetry parameter of EFG is obtained as was observed by Pasternak et al. At the molecular dissociation pressure, the change of the contact density is very small. We conclude that for the solid iodine under pressure the molecular dissociation picture which was proposed by X-ray experiments is consistent with that of the Mössbauer experiments reported by Pasternak et al.

[first-principles calculation, pressure-induced phase transition, diatomic molecular solid, molecular dissociation, hyperfine parameters]
\end{abstract}

\section{Introduction}

The diatomic molecular solids have received much attention from a view point of molecular dissociation and metallization under high pressure. In iodine, among many diatomic molecular solids, these phenomena were firstly observed. The crystal structure remains the same as that of ambient pressure, $D_{2 h}^{18}-C m c a$, up to $21 \mathrm{GPa}$ and hence the metallization at $16 \mathrm{GPa}$ comes from the band overlap [1]. At $21 \mathrm{GPa}$ the molecular dissociation occurs with a 4 percent volume change and the structure transforms to a monatomic body-centered orthorhombic (BCO) one with the space group $D_{2 h}^{25}-\operatorname{Immm}[2]$. At temperatures down to $35 \mathrm{~K}$, the molecular dissociation is also detected at around $21 \mathrm{GPa}$ [3].

New high pressure phases have been reported on the basis of Mössbauer effect measurements at $4 \mathrm{~K}$ above $16 \mathrm{GPa}$ and another high pressure phase above $24 \mathrm{GPa}$ [4]. Mössbauer studies at $75 \mathrm{~K}$ and room temperature [5] confirmed the results. However no evidence of the transitions is found up to $30 \mathrm{GPa}$ by Mössbauer isomer shift [6]. In Ref.4 Pasternak et al. concluded that the molecular phase, $\mathrm{Cmca}$, exists up to $30 \mathrm{GPa}$ based on the relative large asymmetry parameter of the electric field gradient (EFG), that is 0.5 at $30 \mathrm{GPa}$. Their conclusion contradicts the molecular dissociation picture.

\section{Calculation}

In the present study, we carry out the first-principles calculations of electronic states of the solid iodine under high pressure. We use the full-potential linear muffintin-orbital (FP-LMTO) method developed by Savrasov [7] within the local density approximation (LDA). We use four- $\kappa$ for valence electrons: $\kappa^{2}=0.5 \mathrm{Ry}$ is used for $5 s, 5 p$, and $5 d$ states, -0.5 Ry and -1.5 Ry for $5 s$ and $5 p$ states, and -2.5 Ry for $4 d$ state. We use no empty spheres. Inside the muffin-tin spheres (MTS), the scalar-relativistic calculations are performed for valence electrons. The core states are recalculated at each selfconsistent iteration with relativistic effects. Iodine nucleus is treated as uniformly charged sphere, where the radius $R_{n u c}=6 \mathrm{fm}$. Densities, potentials, and the envelope functions inside MTS are expanded up to $l=8$. Densities and potentials at the interstitial region are expanded in the Fourier series with the reciprocal lattice vectors G. About $1700 \mathbf{G}$ vectors per atom are used. The envelope functions at the interstitial region are also expanded in G: $s, p$, and $d$ states are expanded in about 150,200 , and $240 \mathrm{G}$ vectors per atom, respectively. The tetrahedron method improved by Blöchl et al. [8] are used for k-points sampling and $93 \mathbf{k}$-points are sampled for the molecular phase inside the irreducible Brillouin zone. All of these parameters are settled for the total energy convergence within $0.5 \mathrm{mRy}$ per atom and for the contact density $\rho(0)$ within $0.01 a_{0}^{-3}$, where $a_{0}$ is Bohr radius. Throughout all our calculations, the MTS radius is fixed at $2.558 a_{0}$, which is slightly short length from the half of the molecular bond length at zero pressure. The orthorhombic lattice parameters are not optimized and are taken from the X-ray experimental results both the molecular phase and BCO. The pressure values corresponding the each structures are also taken from the experimental results. In the molecular phase, the atomic positions are optimized by the procedure of the frozen phonon calculation.

The EFG at a lattice point is defined as the second derivative of the electrostatic potential $V$ with respect to the Cartesian coordinates at the nuclear site

$$
\Phi_{i j}=\left.\frac{\partial^{2} V}{\partial x_{i} \partial x_{j}}\right|_{n}-\left.\frac{1}{3} \delta_{i j} \nabla^{2} V\right|_{n},
$$

where $\mathrm{EFG}$ is defined as a traceless tensor because there is no interaction of a nuclear quadrupole with a potential caused by $s$ electrons. The electrostatic potential in MTS is written as

$$
V(\mathbf{r})=\sum_{L} V_{L}(r) i^{l} Y_{L}(\hat{\mathbf{r}})
$$

and

$$
\begin{gathered}
V_{L}(r)=\frac{8 \pi}{2 l+1}\left[\frac{1}{r^{l+1}} \int_{0}^{r} x^{l+2} \hat{\rho}_{L}(x) d x\right. \\
\quad+r_{\backslash}^{l} \int_{r}^{S} x^{1-l} \hat{\rho}_{L}(x) d x \\
\left.+r^{l} \sum_{\mathbf{G}} \frac{4 \pi}{G S^{l-1}} Y_{L}^{*}(\hat{\mathbf{G}}) j_{l-1}(G S)[\tilde{n}(\mathbf{G})+\Gamma(\mathbf{G})]\right],
\end{gathered}
$$


where $L$ denotes the combined notation of $(l, m), S$ is the radius of MTS, $\hat{\rho}$ is the $L$ component of the electronic charge density in MTS, and $\tilde{n}$ and $\Gamma$ are the Fourier component of the pseudodensity and the auxiliary density [9], respectively. The electrostatic interaction between a nuclear charge distribution $\rho_{n}\left(\mathbf{r}_{i}\right)$ and $V$ is

$$
E_{i}=\int \rho_{n}\left(\mathbf{r}_{i}\right) V\left(\mathbf{r}_{i}\right) d \mathbf{r}_{i},
$$

where $\rho(\mathbf{r})$ denotes the charge density around the nucleus and $V$ is calculated by Eq.(3). Because the size of nucleus is very small Eq.(4) is written as

$$
E_{i}=\sum_{L} U_{L}^{*} Q_{L}
$$

where

$$
Q_{L}=\sqrt{\frac{4 \pi}{2 l+1}} \int r_{i}^{l} Y_{l m}\left(\hat{\mathbf{r}}_{i}\right) \rho_{n}\left(\mathbf{r}_{i}\right) d \mathbf{r}_{i}
$$

is the nuclear multipole moment and $U$ is coming from the last two terms of Eq.(3):

$$
\begin{aligned}
U_{L} & =2 \sqrt{\frac{4 \pi}{2 l+1}}\left[\lim _{r \rightarrow 0} \int_{r}^{S} x^{1-l} \hat{\rho}_{L}(x) d x\right. \\
& \left.+\sum_{\mathbf{G}} \frac{4 \pi}{G S^{l-1}} Y_{L}^{*}(\hat{\mathbf{G}}) j_{l-1}(G S)[\tilde{n}(\mathbf{G})+\Gamma(\mathbf{G})]\right] .
\end{aligned}
$$

In Eq.(6) the $l=2$ components represent the nuclear quadrupole moments, and the $l=2$ terms of Eq.(7) are the tensor components of EFG. The relation between Eq.(1) and Eq.(7) is as follows [10]:

$$
\begin{aligned}
& \Phi_{x x}=\sqrt{\frac{3}{2}}\left(U_{22}+U_{2-2}\right)-U_{20}, \\
& \Phi_{y y}=-\sqrt{\frac{3}{2}}\left(U_{22}+U_{2-2}\right)-U_{20}, \\
& \Phi_{z z}=2 U_{20}, \\
& \Phi_{x y}=i \sqrt{\frac{3}{2}}\left(-U_{22}+U_{2-2}\right), \\
& \Phi_{y z}=i \sqrt{\frac{3}{2}}\left(U_{21}+U_{2-1}\right), \\
& \Phi_{z x}=\sqrt{\frac{3}{2}}\left(-U_{21}+U_{2-1}\right) .
\end{aligned}
$$

If $\Phi_{i j}$ 's are diagonalized, then an asymmetry parameter, which vary between 0 and 1 , is defined as

$$
\eta=\frac{\Phi_{x x}-\Phi_{y y}}{\Phi_{z z}}
$$

under the condition $\left|\Phi_{z z}\right| \geq\left|\Phi_{y y}\right| \geq\left|\Phi_{x x}\right|$ and $\Phi_{x x}+$ $\Phi_{y y}+\Phi_{z z}=0$.

\section{Results and Discussions}

In the molecular phase, the principal component of EFG gradually decreases with increasing pressure as shown in Fig. 1. The direction of the principal axis of EFG almost lies along the molecular axis. The asymmetry parameter increases with increasing pressure as shown in Fig. 2.

Above 15.3 GPa, however, the direction of the principal axis of EFG lies along the $a$-axis and the sign of the principal component changes as shown in Fig. 1. Such a change is experimentally observed by Pasternak et al. [4]. Above 15.3 GPa, the asymmetry parameter decreases with increasing pressure.

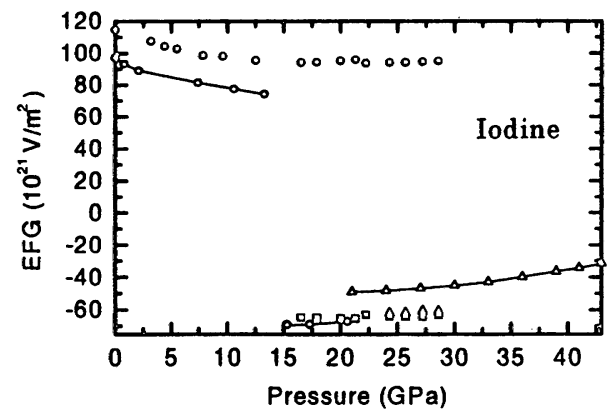

Fig. 1. Pressure dependence of principal component of EFG. Open and filled notations denote calculated and experimental results, respectively.

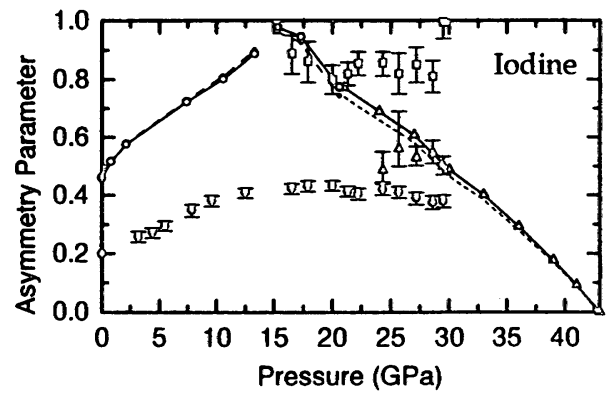

Fig. 2. Pressure dependence of asymmetry parameter of EFG. Open and filled notations denote calculated and experimental results, respectively.

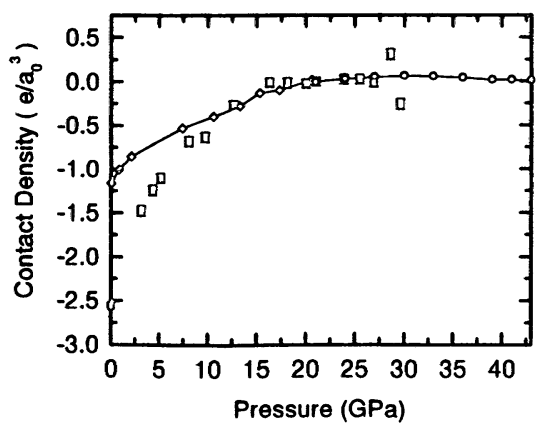

Fig. 3. Pressure dependence of $\rho(0)$. Open and filled notations denote calculated and experimental results, respectively.

At $21 \mathrm{GPa}$, the dissociation pressure, the sign of the principal component does not change. In $\mathrm{BCO}$, the principal component of EFG and the asymmetry parameter 
gradually decreases with increasing pressure. The asymmetry parameter reaches zero at $43 \mathrm{GPa}$, the secondorder transition pressure to the body-centered tetragonal (BCT) structure. As shown in Fig. 2 the large asymmetry parameter, i.e. 0.49 at $30 \mathrm{GPa}$, is obtained as observed by Pasternak et al. [4].

In the molecular phase, the change of the direction of the principal axis is caused by the change of the electronic contribution due to the band overlap and the gradual decrease of interatomic distances with increasing pressure. In $\mathrm{BCO}$, the large asymmetry parameter is caused by the lattice distortion which is due to the band Jahn-Teller effect [11].

As shown in Fig. 3, $\rho(0)$ increases with increasing pressure in the molecular phase. At the dissociation pressure, the change is only $-0.02 \mathrm{e} / a_{0}^{3}$. In $\mathrm{BCO}, \rho(0)$ changes negligibly.

The vanishingly small change in $\rho(0)$ at high pressure is attributed to the cancellation between the contributions of $5 s$ - and $5 p$-electrons [11]. Such a change could not be detected by the isomer shift measurement [6].

In this study, hyperfine parameters of solid iodine under high pressure are studied theoretically. We conclude that the results of Mössbauer spectroscopy for solid iodine under high pressure consist with the molecular dissociation picture.

\section{Acknowledgements}

We would like to thank sincerely Dr. S. Y. Savrasov for providing us with the FP-LMTO programs. We would like to express our gratitude to Dr. H. Fujihisa for providing us with his experimental data prior to publication.

\section{References}

*Present address: Advanced Technology Research Laboratories, Central Research Institute, Mitsubishi Materials Corporation, 1-297 Kitabukuro-cho, Omiya, Saitama 330, Japan.

[1] O. Shimomura, K. Takemura, Y. Fujii, S. Minomura, M. Mori, Y. Noda, and Y. Yamada, Phys. Rev. B18, 715 (1978).

[2] K. Takemura, S. Minomura, O. Shimomura, and Y. Fujii, Phys. Rev. Lett. 45, 1881 (1980).

[3] H. Fujihisa, Y. Fujii, K. Hase, Y. Ohishi, N. Hamaya, K. Tsuji, K. Takemura, O. Shimomura, H. Takahashi, and T. Nakajima, High Pressure Res. 4, 330 (1990).

[4] M. Pasternak, J. N. Farrell, and R. D. Taylor, Phys. Rev. Lett. 58, 575 (1987).

[5] M. Pasternak, J. N. Farrell, and R. D. Taylor, Phys. Rev. Lett. 59, 945 (1987).

[6] M. Pasternak, J. N. Farrell, and R. D. Taylor, Solid State Commun. 61, 409(1987).

[7] S. Y. Savrasov, Phys. Rev. B 54, 16470 (1996).

[8] P. E. Blöchl, O. Jepsen, and O. K. Andersen, Phys. Rev. B 49, 16233 (1994).

[9] K. H. Weyrich, Phys. Rev. B 37, 10269 (1988).

[10] E. Matthias, W. Schneider, and R. M. Steffen, Ark. Fys. 24, 97 (1963).

[11] K. Yamaguchi and H. Miyagi, Solid State Commun. 99, 89 (1996). 